\title{
Infiltration of a mixture of different immune cells may be related to molecular profile of differentiated thyroid cancer
}

\section{Dear Editor}

Pathologists have long recognized that some tumors are densely infiltrated by cells of both innate and adaptive arms of the immune system, thereby reflecting inflammatory conditions arising in non-neoplastic tissues (Dvorak 1986, Hanahan \& Weinberg 2011). Such infiltration may reflect an effort of the immune system attempting to eradicate neoplastic cells. Conversely, there is an increasing amount of evidences for antitumor responses, resulting from a pressure on the tumor to evade immune destruction (Hanahan \& Weinberg 2011). Tumor evolution and consequent patient outcomes may depend on this complex interaction between immune system and tumor cells.

The immune responses against differentiated thyroid carcinomas (DTC) have also been extensively studied (Modi et al. 2003). CD4+ T cells are central to the successful orchestration of this immune response. Naive CD4 + T cells differentiate into one of at least four functionally distinct fates, such as Th1, Th2, Th17, and regulatory T cells (Tregs). In general, Tregs are identified as FOXP3 + lymphocytes and are thought to contribute to tumor-specific T-cell tolerance (Zhou \& Levitsky 2007). Th17 cells were recently described but, despite their important role in host protection against infectious pathogens and in the pathogenesis of various inflammatory and autoimmune diseases, their prevalence in human cancer is still under investigation (Su et al. 2010).

Antitumor immune response is thought to be related to tumor antigenicity. In fact, a correlation between the protein expression profile and immune cell infiltration in DTC was observed (Bruland et al. 2009). Some researchers have suggested that MUC1, NIS (SLC5A5), ATM, PTEN, and CD56 (NCAM1) expression might indicate tumor differentiation and tumor progression, demonstrating tumor antigenicity (Larson et al. 2007, El Demellawy et al. 2008, Morari et al. 2010). However, whether these markers are really associated with DTC immune response is still not clear.
In order to examine the putative association between tumor molecular profile and pattern of infiltration of tumor-infiltrating lymphocytes (TIL), tumor-associated macrophages (TAM), and myeloidderived suppressor cells (MDSCs), we investigated 398 patients whose tissue samples were maintained in the tissue bank of the institution. Thyroid carcinoma was diagnosed in 266 patients: 253 with papillary thyroid carcinomas (PTC) including 153 classical form, 80 PTC follicular variants, 20 PTC tall cell variants, and 13 with follicular carcinomas (FC): seven minimally invasive and six frankly invasive. We also obtained 132 normal or benign thyroid tissues, including 18 normal tissues, 58 nodular goiters, and 56 follicular adenomas (FA).

Patients' clinical information was obtained from their files. Aggressiveness at diagnosis was ascertained using the TNM (tumour, node, metastasis) and stage classification system for DTC. Patients underwent periodic total body scans, serum TSH, and thyroglobulin $(\mathrm{Tg})$ measurements according to a standard protocol for a period of 12-298 (43.50 \pm 33.29$)$ months, $\mathrm{Mo}=21$ months. Patients presenting high nonstimulated serum $\mathrm{Tg}$ levels $(>2 \mathrm{ng} / \mathrm{dl}$ and, more recently, $\mathrm{Tg}$ $>1 \mathrm{ng} / \mathrm{dl}$ using a sensitive assay) were submitted to a thorough image search. We defined tumors as persistent/recurrent and/or presenting long distance metastasis, according to the aforementioned parameters.

Formalin-fixed paraffin-embedded tissues from all 398 cases were reviewed for diagnostic confirmation and used to build a tissue microarray (TMA, Beecher Instruments, Silver Springs, MD, USA) for immunohistochemical (IHC) analysis. We obtained four tumor tissue cores from each case. Two spots were chosen from representative areas of the lesion based on the greater leukocyte infiltration while two other spots were chosen from areas free of leukocyte infiltration. The IHC markers were divided into two groups: a) immune cell markers and b) tumor markers. Immune cell markers included TIL and TAM subsets, such as CD3, CD4, CD8, CD20, and CD68, always evaluated in intraepithelial infiltrating cells located within cancer 
cell nests, as showed in Fig. 1, panel I. Tumor markers included CD56, NIS, MUC1, PTEN, ATM, and B7H1 (CD274). IHC procedure was held as described previously running positive and negative controls in the same batch of reactions (Morari et al. 2010).

Infiltration of Th17 and MDSCs was assessed by immunofluorescence. CD4, IL17, CD33, and CD11b expressions were analyzed as described previously (Bertelli et al. 2006). The secondary antibodies were FITC or a rhodamine conjugate. The images were obtained using a confocal laser microscopy (LSM510; Zeiss, New York, NY, USA). Analysis and documentation of results were performed using a Leica FW 4500 B microscope.

The slides were scored independently by two pathologists (J Vassallo and F A Soares), both blinded to tumor features. Cells were considered positive for IHC markers when a clear-cut brown staining was observed in the typical cellular localization. Th17 was defined as those cells positive for immunofluorescence markers when a clear CD4 + and IL17 + co-localization was observed in the typical cellular localization, as shown in Fig. 1, panel II. Likewise, MDSCs were defined as those cells positive for immunofluorescence markers when a clear $\mathrm{CD} 33+$ and $\mathrm{CD} 11 \mathrm{~b}+$ colocalization was observed in the typical cellular localization, as shown in Fig. 1, panel III. An individual evaluation of immune cell markers was completed for each tissue spot, estimating the number of positive cells per TMA spot considering an approximate area of $0.79 \mathrm{~mm}^{2}$. The cases were grouped into categories for statistical analysis: 0 (no positive cell), $1+$ (up to ten positive cells per spot), and $2+$ (ten or more positive cells per spot).

Statistical analysis was carried out using the Winstat Software (Cambridge, MA, USA). Nonparametric analysis was performed using the $\chi^{2}$ or Fisher's exact test as indicated. A multivariate logistic regression model was applied using tumor type as a dependent variable, protein expression, and clinical risk factors, including gender and age as explicative variables. Mann-Whitney $U$ tests were used to compare continuous or arranged measures between two groups whose variable did not present Gaussian distribution; Kruskal-Wallis test was used to compare three or more groups whose variable did not present Gaussian distribution. The $T$-test or ANOVA test was used for variables that presented Gaussian distribution, comparing two, three, or more groups respectively. All tests were conducted at a 0.05 significance level.

As expected, most $(83.66 \%)$ of our patients were females. Individuals with benign $(49.29 \pm 15.18$ years old) and malignant $(43.66 \pm 15.91$ years old) thyroid lesions presented similar ages at diagnosis. DTC patients were classified according to the pathological TNM (pTNM) staging system (Shaha 2007) as pTNM I (157 cases), II ( 28 cases), III (40 cases), and IV (41 cases). Ninety tumors were classified as encapsulated and 176 as nonencapsulated; 118 tumors were multifocal and 148 unifocal; 108 presented extrathyroidal invasion, whereas 158 tumors did not. Forty-eight patients (18.04\%, including ten dead-of-disease) presented recurrence, whereas 185 evolved free of disease $(69.55 \%)$. Thirty-three patients who could not be classified into one of these two groups were further excluded from any analysis involving outcome.

Macrophages were not found in normal thyroid tissue. TAM was more frequent in malignant $(82.11 \%)$ than in benign tissues $(33.91 \% ; P<0.0001)$. As depicted in Table 1, TAM was more frequent in tumors expressing MUC1 $(P<0.0001)$, ATM $(P<0.0001)$, and B7H1 $(P=0.0035)$.

None of the normal thyroid tissues were CD3+, only one nodular goiter, and four follicular adenomas. Conversely, CD3 + infiltration occurred in $59.17 \%$ of malignant tissues $(P<0.0001)$, at similar rates in PTC and follicular thyroid carcinomas tissues $(P=0.8596)$. $\mathrm{CD} 3+$ was more frequent in FTC than in FA cases $(P<0.0001)$, and in follicular variant of papillary thyroid carcinomas than in FA cases $(P<0.0001)$, helping to characterize follicular-patterned lesions, but it did not differentiate FVPTC from FC $(P=0.5309)$. Infiltration of $\mathrm{CD} 3+$ lymphocytes also correlated with MUC1, CD4 +, CD8 +, CD20 +, and TAM (Table 1).

None of the normal thyroid tissues was CD4 +. Only two nodular goiters $(3.44 \%)$ and one FA (1.78\%) displayed CD4 + lymphocytes, in contrast to 41 out of the malignant samples $(15.41 \%)$, hence distinguishing benign $(2.63 \%)$ and malignant nodules $(15.41 \%$; $P=0.0010)$. CD4 + lymphocyte infiltration was more frequent in tumors positive for B7H1, MUC1, ATM, and CD56, presenting TAM, CD3+, CD8+, and CD20+ lymphocytes (Table 1).

Malignant thyroid tissues showed CD8 + lymphocytes more frequently $(46.12 \%)$ than goiter samples $(37.5 \%)$ and FA (37.74\%). However, 17 normal thyroid tissues presented evident CD8 + lymphocyte infiltration and one sample displayed only a few scattered cells spread throughout the tissue. Intense $\mathrm{CD} 8+$ lymphocytic infiltration occurred more frequently in malignant $(23.29 \%)$ than in benign $(3.53 \%)$ thyroid tissues $(P<0.0001)$. CD8 + lymphocyte infiltration was more frequent in tumors expressing MUC1, ATM, PTEN, NIS, CD56, B7H1, and showing $\mathrm{TAM}, \mathrm{CD} 3+, \mathrm{CD} 4+$, and $\mathrm{CD} 20+$ lymphocytes (Table 1). 


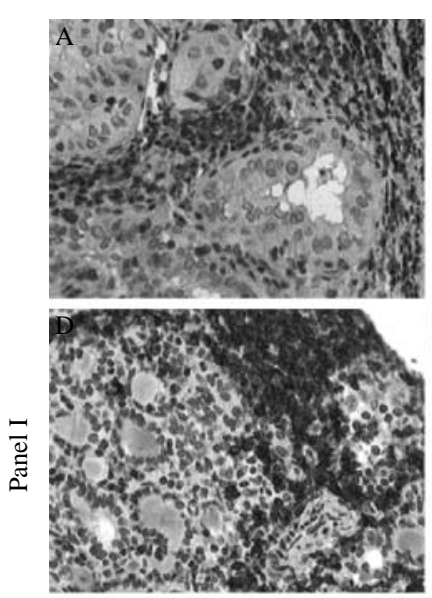

DAPI
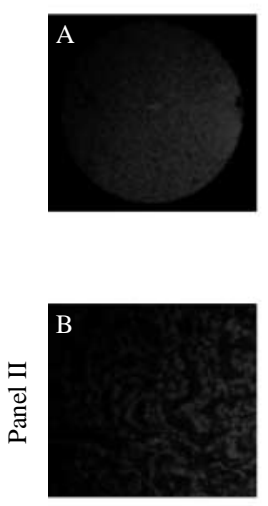

DAPI
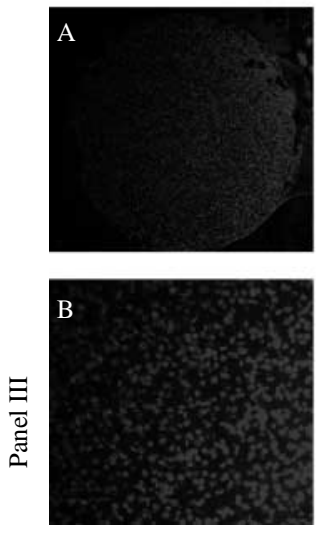
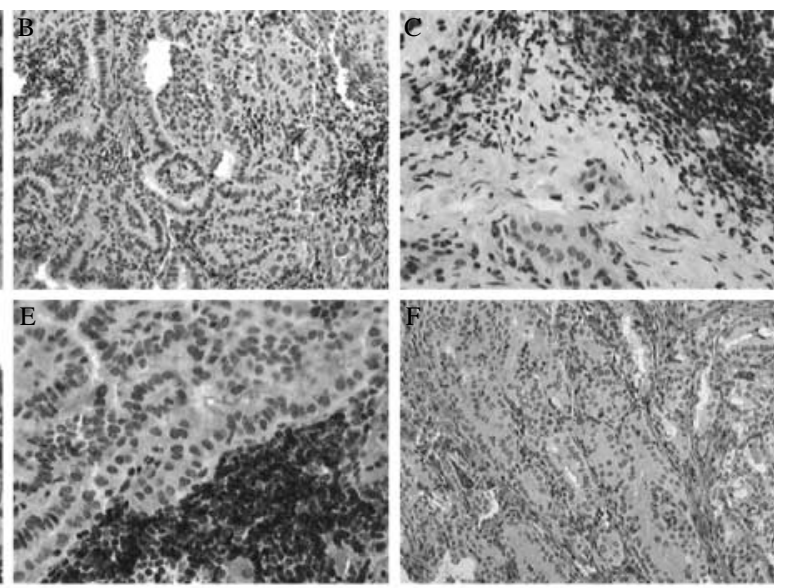

IL17
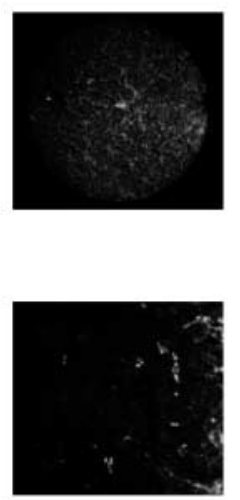

$\mathrm{CD} 11 \mathrm{~b}$
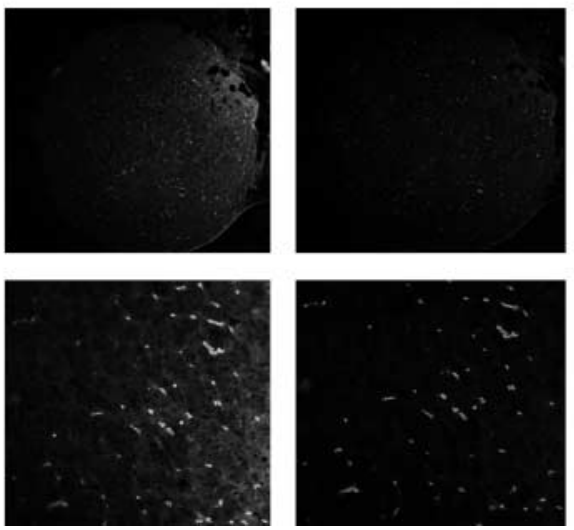
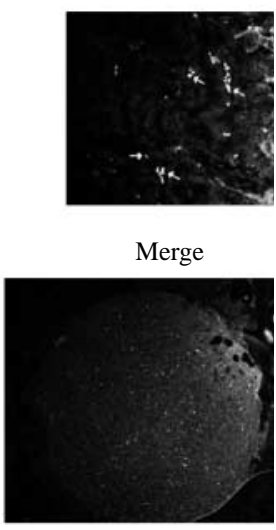

Merge
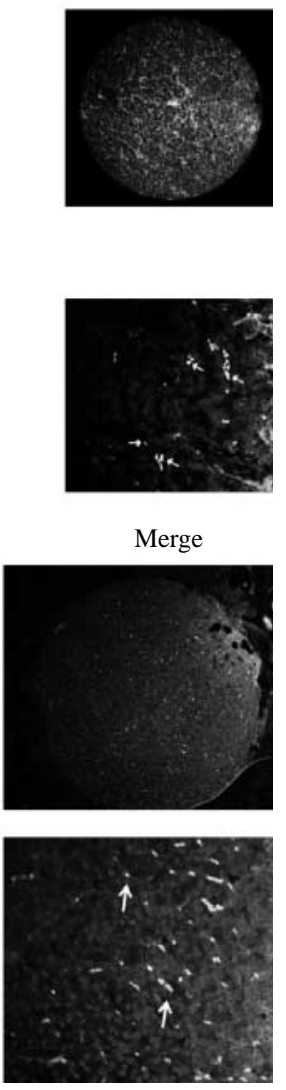

Figure 1 (Panel I) Immune cells in papillary thyroid carcinomas. All images correspond to immuohistochemical reactivity for each of the markers, as evidenced by brown staining in the membrane of lymphocytes. (A) Infiltration of CD3+ lymphocytes $(400 \times)$, which is a pan-T marker. (B) Infiltration of CD8 + T lymphocytes. CD8 + (cytotoxic T) lymphocytes are found as a diffuse infiltration between the papillae. (C) Infiltration of CD4 + (helper T) lymphocytes infiltrating PTC lesion $(400 \times)$. (D and E) Infiltration of CD20+

lymphocytes (B lymphocytes). (F) Infiltration of CD68 + cells (macrophages at 50 X). (Panel II) CD4+/IL17+ lymphocytes infiltrate PTC demonstrated by immunofluorescence colocalization of CD4+ and IL17+ markers. (Panel A) TMA images for CD4+/IL17+ cells in PTC (magnification: $50 \times$ ). (Panel B) PTC with infiltration of CD4 +/IL17+ lymphocytes (magnification: $400 \times$ ). White arrows indicate CD4+/IL17+ (Th17) lymphocytes. The nuclei of PTC cells were characterized by their typical appearance and were easily distinguished from infiltrating lymphocytes. CD4+/IL17+ lymphocytes were found as single cells or multicellular clusters within the tumor. (Panel III) CD33+/CD11b + cells infiltrate PTC demonstrated by immunofluorescence colocalization of CD33+ and CD11b + markers. (Panel A) TMA images for CD33+/CD11b + cells in PTC (magnification: $50 \times$ ). (Panel B) PTC with infiltration of CD33 +/CD11b + cells (magnification: 400X). White arrows indicate CD33+/CD11b + myeloid-derived suppressor cells (MDSCs). CD33+/CD11b + cells were found as diffuse or multicellular clusters scattered within the tumor. 


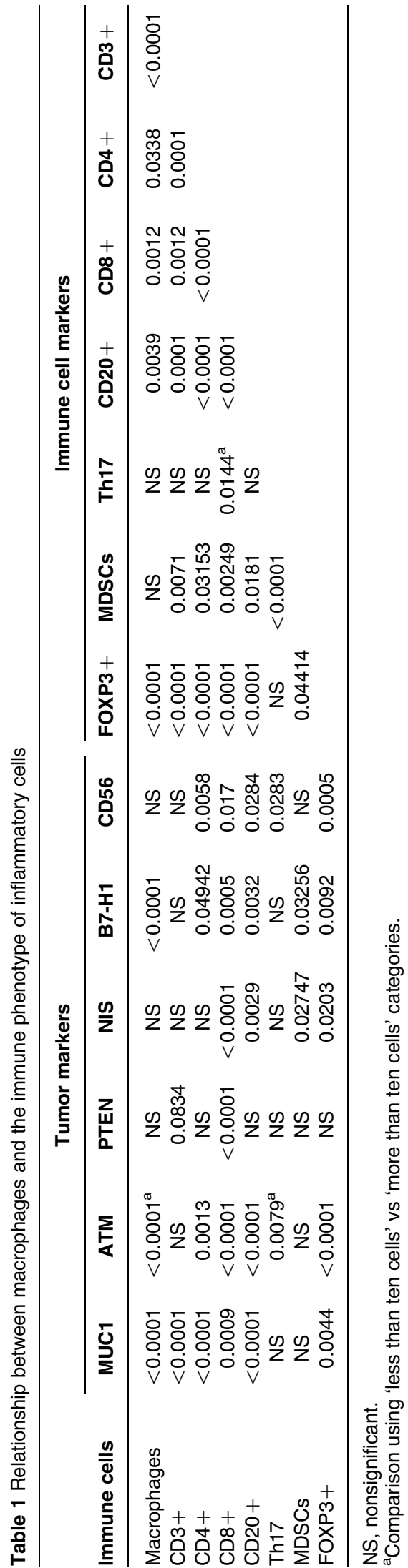

None of the normal thyroid tissues or the FA examined and only two nodular goiters displayed $\mathrm{CD} 20+$ lymphocytic infiltration in contrast to 46 $(23.11 \%)$ of malignant tissues $(P<0.0001)$. CD20+ lymphocytes were more frequent in PTC $(27.33 \%)$ than in FTC $(5.26 \% ; P=0.0024)$. CD20+ was more frequent in tumors expressing MUC1, ATM, NIS, CD56; TAM, CD3+, CD4+, and CD8 + lymphocytes infiltration, as shown in Table 1.

CD4+/IL17+ lymphocytic infiltration was more frequent in malignant tissues $(98.22 \%)$ than in nonmalignant tissues $(87.23 \% ; P=0.0004)$. CD4+ $/$ IL17 + lymphocyte infiltration was more frequent in tumors expressing ATM, CD56, and CD8 + lymphocytes as shownin Table 1. Figure 1, panel II, shows a representative immunohistochemistry of $\mathrm{CD} 4+$ / IL17+ lymphocytic infiltration in PTC tissue.

None of the normal thyroid tissues, goiters, FA, or FC presented FOXP3 + lymphocytes. Only 14.5\% PTC were FOXP3 +. FOXP3 + lymphocytes were more frequent in tumors expressing MUC1, ATM, NIS, CD56, and B7H1 and in infiltration of macrophages, $\mathrm{CD} 3+, \mathrm{CD} 4+, \mathrm{CD} 8+$, and CD20 + lymphocytes (Table 1).

We further investigated the association between infiltration of MDSCs and the molecular profile of our DTC cases. Indeed, tumors positive for B7H1 were frequently enriched with MDSCs $(P=0.0455)$. Infiltration of MDSCs was accompanied by infiltration of $\mathrm{CD} 3+(P=0.0284)$ and $\mathrm{CD} 8+(P=0.0050)$ lymphocytes. Tumors that presented MDSCs were frequently positive for NIS $(P=0.0439)$.

In summary, our data demonstrated that immune cells infiltrate malignant tissues more often than benign lesions, suggesting an immune reaction of the organism against transformed cells. In addition, immune cell infiltration was closely associated with the IHC profile of the DTC specimens examined.

Some proteins involved in the pathogenesis of DTC might account for part of the immune reaction as differentiation antigens, as their expression is significantly associated with the inflammation process. MUC1, NIS, and CD56 were found as common markers of antitumor immune response. There are evidences that these markers may act as autoantigens (Moore et al. 2004, El Demellawy et al. 2009).

We also found PTEN and ATM expression associated with immune cell infiltration, supporting the idea that these proteins might enhance tumor skills to induce an immune response (Noh et al. 2009). Endorsing this assumption, it has been demonstrated that loss of ATM is associated with immune resistance (Gomez et al. 2008). Also, B7H1 is classically engaged 
in immune evasion. In fact, cancer cell expression of costimulatory molecule B7-H1 has been implicated as a potent inhibitor of T-cell-mediated antitumoral immunity (Thompson et al. 2005). However, association between $\mathrm{B} 7 \mathrm{H} 1$ expression in tumor cells and infiltration of different immune cells may rise the idea that these immune cells are not functionally activated. More studies are warranted to figure this question.

In conclusion, we observed an infiltration of a mixture of different immune cells in DTC. The fact that these immune cells are correlated with each other suggests that a complex immune network may be elicited by the cancerous thyroid follicular cell. Also, we demonstrated that the immune response may be influenced by the status of DTC tumor antigenicity. In addition, immune cell infiltration profile helped to characterize specific tumor histotypes.

Lucas Leite Cunha ${ }^{1}$ Elaine Cristina Morari ${ }^{1}$ Ana Carolina Trindade Guihen ${ }^{1}$ Daniela Razolli ${ }^{2}$ Renê Gerhard ${ }^{3}$ Suely Nonogaki ${ }^{4}$ Fernando Augusto Soares ${ }^{5}$ José Vassallo ${ }^{6}$

Laura Sterian Ward ${ }^{1}$

${ }^{1}$ Laboratory of Cancer Molecular Genetics, Faculty of Medical Sciences, University of Campinas (Unicamp), PO Box 6111, 126 Tessalia Vieira de Camargo Street, Campinas, São Paulo, Brazil

${ }^{2}$ Laboratory of Cell Signaling,

Faculty of Medical Sciences, University of Campinas (Unicamp), 420 Carlos Chagas Street, Campinas,

São Paulo, Brazil

${ }^{3}$ Department of Radiology, Faculty of Medicine, University of São Paulo (USP), 255 Dr Enéas de Carvalho Aguiar Avenue, São Paulo, Brazil

${ }^{4}$ Adolfo Lutz Institute, 355 Doutor Arnaldo Avenue, São Paulo, Brazil

${ }^{5}$ Department of Pathology,

A. C. Camargo Cancer Hospital, 211 Antonio Prudente Street, São Paulo, São Paulo, Brazil

${ }^{6}$ Laboratory of Investigative and Molecular Pathology, CIPED, FCM-Unicamp, and Department of Pathology,

\section{Hospital do Cancer A C Camargo, São Paulo, Brazil}

(Correspondence should be addressed to L S Ward; Email: ward@fcm.unicamp.br)

\section{Declaration of interest}

The authors declare that there is no conflict of interest that could be perceived as prejudicing the impartiality of the research reported.

\section{Funding}

This research did not receive any specific grant from any funding agency in the public, commercial or not-for-profit sector.

\section{Author contribution statement}

L L Cunha contributed in conception and design, collection and assembly of data, data analysis and interpretation, manuscript writing, and final approval of manuscript. E C Morari and A C T Guihen contributed in collection and assembly of data and final approval of manuscript. D Razolli, R Gerhard, and $\mathrm{S}$ Nonogaki contributed in data analysis and interpretation, histopathological analysis, and final approval of manuscript. F A Soares and J Vassallo contributed histopathological analysis and final approval of manuscript. L S Ward contributed in conception and design, data analysis and interpretation, manuscript writing, and final approval of manuscript.

\section{Acknowledgements}

We thank Etna Macário and Marcella Lima de Souza for their valuable suggestions and insights. This study was supported by the State of São Paulo Research Foundation (FAPESP, n. 2009/18362-0). Funding source had no involvement in study design; in the collection, analysis, and interpretation of data; in the writing of the report; and in the decision to submit the paper for publication.

\section{References}

Bertelli DF, Araujo EP, Cesquini M, Stoppa GR, Gasparotto-Contessotto M, Toyama MH, Felix JV, Carvalheira JB, Michelini LC, Chiavegatto S et al. 2006 Phosphoinositide-specific inositol polyphosphate 5-phosphatase IV inhibits inositide trisphosphate accumulation in hypothalamus and regulates food intake and body weight. Endocrinology 147 5385-5399. (doi:10.1210/en.2006-0280)

Bruland O, Fluge O, Akslen LA, Eiken HG, Lillehaug JR, Varhaug JE \& Knappskog PM 2009 Inverse correlation between PDGFC expression and lymphocyte infiltration in human papillary thyroid carcinomas. BMC Cancer 9 425. (doi:10.1186/1471-2407-9-425) 
Dvorak HF 1986 Tumors: wounds that do not heal. Similarities between tumor stroma generation and wound healing. New England Journal of Medicine 315 1650-1659. (doi:10.1056/NEJM198612253152606)

El Demellawy D, Nasr A \& Alowami S 2008 Application of CD56, P63 and CK19 immunohistochemistry in the diagnosis of papillary carcinoma of the thyroid. Diagnostic Pathology 3 5. (doi:10.1186/1746-1596-3-5)

El Demellawy D, Nasr AL, Babay S \& Alowami S 2009 Diagnostic utility of CD56 immunohistochemistry in papillary carcinoma of the thyroid. Pathology, Research and Practice 205 303-309. (doi:10.1016/j.prp.2008.11.011)

Gomez GG, Hickey MJ, Tritz R \& Kruse CA 2008 Immunoresistant human glioma cell clones selected with alloreactive cytotoxic $\mathrm{T}$ lymphocytes: downregulation of multiple proapoptotic factors. Gene Therapy \& Molecular Biology 12 101-110.

Hanahan D \& Weinberg RA 2011 Hallmarks of cancer: the next generation. Cell 144 646-674. (doi:10.1016/j.cell. 2011.02.013)

Larson SD, Jackson LN, Riall TS, Uchida T, Thomas RP, Qiu S \& Evers BM 2007 Increased incidence of welldifferentiated thyroid cancer associated with Hashimoto thyroiditis and the role of the PI3k/Akt pathway. Journal of the American College of Surgeons 204 764-773 discussion 773-775. (doi:10.1016/j.jamcollsurg.2006.12.037)

Modi J, Patel A, Terrell R, Tuttle RM \& Francis GL 2003 Papillary thyroid carcinomas from young adults and children contain a mixture of lymphocytes. Journal of Clinical Endocrinology and Metabolism 88 4418-4425. (doi:10.1210/jc.2003-030342)
Moore A, Medarova Z, Potthast A \& Dai G 2004 In vivo targeting of underglycosylated MUC-1 tumor antigen using a multimodal imaging probe. Cancer Research 64 1821-1827. (doi:10.1158/0008-5472.CAN-03-3230)

Morari EC, Silva JR, Guilhen AC, Cunha LL, Marcello MA, Soares FA, Vassallo J \& Ward LS 2010 Muc-1 expression may help characterize thyroid nodules but does not predict patients' outcome. Endocrine Pathology 21 242-249. (doi:10.1007/s12022-010-9137-4)

Noh KH, Kang TH, Kim JH, Pai SI, Lin KY, Hung CF, Wu TC \& Kim TW 2009 Activation of Akt as a mechanism for tumor immune evasion. Molecular Therapy 17 439-447. (doi:10.1038/mt.2008.255)

Shaha AR 2007 TNM classification of thyroid carcinoma. World Journal of Surgery 31 879-887. (doi:10.1007/ s00268-006-0864-0)

Su X, Ye J, Hsueh EC, Zhang Y, Hoft DF \& Peng G 2010 Tumor microenvironments direct the recruitment and expansion of human Th17 cells. Journal of Immunology 184 1630-1641. (doi:10.4049/jimmunol. 0902813)

Thompson RH, Gillett MD, Cheville JC, Lohse CM, Dong H, Webster WS, Chen L, Zincke H, Blute ML, Leibovich BC et al. 2005 Costimulatory molecule B7-H1 in primary and metastatic clear cell renal cell carcinoma. Cancer 104 2084-2091. (doi:10.1002/cncr. 21470)

Zhou G \& Levitsky HI 2007 Natural regulatory T cells and de novo-induced regulatory $\mathrm{T}$ cells contribute independently to tumor-specific tolerance. Journal of Immunology 178 2155-2162. 